\title{
APROVEITE A SUA PASSAGEM E VIAJE PELO MUNDO DA LEITURA: UMA ANÁLISE DAS BIBLIOTECAS TRANSCOL DE VILA VELHA POR MEIO DA FERRAMENTA SERVQUAL
}

ENJOY YOUR PASSAGE AND JOURNEY FOR THE WORLD OF READING: AN ANALYSIS OF THE TRANSCOL LIBRARIES OF VILA VELHA THROUGH THE SERVQUAL TOOL

APROVECHE SU PASO Y VIAJE POR EL MUNDO DE LA LECTURA: UN ANÁLISIS DE LAS BIBLIOTECAS TRANSCOL DE VILA VELHA POR MEDIO DE LA HERRAMIENTA SERVQUAL

Sandra Maria Souza de Carvalho ${ }^{1}$

Marcelo Calderari Miguel ${ }^{2}$

Ana Claudia Borges Campos ${ }^{1}$

${ }^{1}$ Universidade Federal do Espírito Santo

${ }^{2}$ Universidade Federal de Minas Gerais

\section{Correspondência}

${ }^{1}$ Sandra Maria Souza de Carvalho

Universidade Federal do Espírito Santo

Vitória, ES

E-mail: sandramsc@hotmail.com

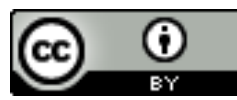

JITA: CA. Use studies.

Submetido em: $15 / 12 / 2017$

Aceito em: 23/04/2018

Publicado em: 27/08/2018 
RESUMO: Avaliar e reavaliar a qualidade de ajustar as necessidades de sua comunidade são atividades necessárias para qualquer biblioteca. Este estudo utiliza a métrica Servqual - de Parasuraman, Zeithaml e Berry (1985), para analisar as expectativas e percepção dos associados da Bibiblioteca Transcol em relação aos serviços ofertados pelos módulos bibliotecários nos términos de transporte públicos do município de Vila Velha. Este método propõe a analise da matriz importância \& desempenho para a gestão das bibliotecas públicas em relação às cinco dimensões de qualidade: confiança, tangibilidade, sensibilidade, segurança e empatia. A metodologia desta investigação tem caráter descritivo com o uso da técnica de incidente crítico. Os resultados obtidos indicam que os associados da biblioteca atribuem maior importância à dimensão 'segurança' no que refere à prestação de serviços em informação.

PALAVRAS-ChavE: Estudo de usuário. Serviço comunitário de informação. Servqual.

ABSTRACT: Evaluating and reassessing the quality of adjusting the needs of your community are necessary activities for any library. This study uses the Metrics Servqual - of Parasuraman, Zeithaml and Berry (1985), to analyze the expectations and perception of the members of the Transcol Library in relation to the services offered by the librarian modules in the public transportation terms of the municipality of Vila Velha. This method proposes the analysis of the matrix importance \& performance for the management of public libraries in relation to the five dimensions of quality: reliability, tangibility, sensitivity, assurance and empathy. The methodology of this investigation has a descriptive character with the use of the technique of critical incident. The results indicate that the members of the library attach greater importance to the 'assurance' dimension in relation to the provision of information services.

KEYWORDS: User study. Community information service. Servqual.

RESUMEN: Evaluar y reevaluar la calidad del ajuste de las necesidades de su comunidad son actividades necesarias para cualquier biblioteca. Este estudio utiliza Metrics Servqual - de Parasuraman, Zeithaml y Berry (1985), para analizar las expectativas y la percepción de los miembros de la Biblioteca Transcol en relación con los servicios ofrecidos por los módulos bibliotecarios en los términos de transporte público del municipio de Vila Velha. Este método propone el análisis de la importancia y el rendimiento de la matriz para la gestión de las bibliotecas públicas en relación con las cinco dimensiones de la calidad: confianza, tangibilidad, sensibilidad, seguridad y empatía. La metodología de esta investigación tiene un carácter descriptivo con el uso de la técnica del incidente crítico. Los resultados indican que los miembros de la biblioteca otorgan mayor importancia a la dimensión de "seguridad" en relación con la provisión de servicios de información.

Palabrasclave: Estudio de usuario. Servicio comunitario de información. Servqual. 


\section{A MINUTOS DE QUALQUER DESTINO... EIS A BIBLIOTECA}

"Tudo para todos é exatamente o que a biblioteca pública deve ser" (TOTTERDELL apud FONSECA, 2007, p. 68). E superado o velho arquétipo de depositório de saber, lugar de silêncio e recinto da conservação, a 'nova biblioteca pública' surge para subsidiar práticas de letramento informacional e inclusão social. Portanto, a biblioteca pública moderna existe para "facilitar o acesso a informação, promover o gosto pela leitura e pela cultura e fazê-lo da forma mais agradável, cativando, seduzindo, atraindo o utilizador" (BAGANHA, 2004, p. 93).

Moro, Estabel e Behr (2014) esclarecem que biblioteca, visando o acesso às novas aprendizagens e informações, torna-se presença significativa em uma sociedade democrática que segue os preceitos da sociedade de informação e do conhecimento. Segundo estes autores, a qualidade dos serviços da biblioteca está voltada para identificar as necessidades do usuário, partindo da percepção e expectativas que estes apresentam em relação ao funcionamento e à prestação de serviços e recursos informacionais.

Nessa mesma direção, Nunes (2007, p. 49) reconhece que o objetivo da biblioteca pública é prestar serviços de acesso ao conhecimento e promover atividades que fortaleçam a ampliação do letramento informacional, proporcionando acesso a produtos culturais e artísticos em geral, bem como aos testemunhos da memória e da identidade local. Decerto a biblioteca pública "obriga-se à prestação de serviços de educação e cultura que satisfaçam as necessidades dos diversos grupos de utilizadores, definidos por uma ampla heterogeneidade, que é por sua vez o reflexo da heterogeneidade do tecido social" (NUNES, 2007, p. 49).

Assim, nos dias atuais, as bibliotecas incluem novas tecnologias de informação e comunicação (NTCIs); e estas facilitam monitorar o desempenho institucional dos serviços e produtos que são ofertados. Logo os instrumentos de avaliação são elementares nessa ambiência para "fundamentar decisões, planejar, avaliar e analisar cenários e, ainda, oferecer serviços que atendam de forma objetiva ás necessidades informacionais dos usuários" (MORO; ESTABEL; BEHR, 2014, p. 58).

Portanto as bibliotecas públicas, como instrumento social de informação e conhecimento, devem implementar e utilizar serviços básicos, tecnológicos e profissionais que permitam aos seus interagentes a aprendizagem permanente e o uso autônomo da informação (FREITAS; REGEDOR, 2007). Desta forma, o papel essencial da biblioteca pública perpassa pelo letramento informacional, e além disso oportuniza a aprendizagem ao longo da vida, o combate a infoexclusão e a prática leitora. E sobre esse enfoque evidencia-se que:

[...] desde o contexto de seu surgimento, até hoje, à biblioteca pública confere-se um papel abrangente. Ela deve atender a toda a comunidade indiscriminadamente como mostram as proposições do manifesto da UNESCO sobre Bibliotecas Públicas' (1994), que define como 'porta de acesso local à informação'[...] [mas são]

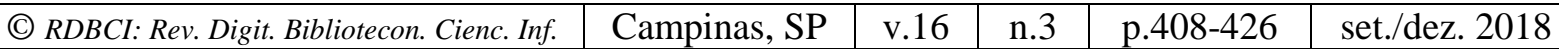


os serviços é que vão conferir à biblioteca sua dinâmica, sua capacidade de transpor a métrica e estabilidade de seus acervos, permitido a concretização de sua 'função social' [...] (RASCHE; VARVAKIS, 2006, p. 128-137).

Deste modo, o projeto intitulado 'BibTranscol' (Biblioteca Transcol) sustentou-se através das ações voltadas à instalação de módulos de locação de livros nos terminais do Sistema Integrado de Transporte Coletivo Urbano Municipal e Intermunicipal de Passageiros da Região Metropolitana da Grande Vitória (TRANSCOL). Os interessados se cadastram nas próprias bibliotecas e podem locar gratuitamente as obras do acervo.

O presente artigo pretende abordar o seguinte problema: quais as 'percepções e expectativas' da comunidade usuária das Bibliotecas Transcol (BibTranscol) de Vila Velha sobre o serviço ofertado. O objetivo geral da pesquisa é averiguar a expectativa e a percepção dos associados da BibTranscol quanto a qualidade dos serviços prestados nos terminais TRANSCOL de São Torquato, Ibes, Itaparica e Vila Velha - tendo como foco o olhar da clientela destes serviços. Em tempos atuais a importância atribuída à qualidade em serviços vem acentuando, o que pode ser observado no crescente número de estudos sobre a gestão da qualidade e seus recorrentes enfoques em estudos organizacionais; afinal, as instituições buscam a entropia negativa, ou seja, sobreviver - e transformar para favorável a imagem da instituição junto a seus públicos prioritários (MIGUEL; FREIRE, 2016).

A metodologia utiliza uma releitura da abordagem teórico-metodológica Servqual (PARASURAMAN; ZEITHAML; BERRY, 1985). A métrica Servqual foi idealizada do conceito de gaps, obtidos pela comparação da expectativa com a percepção que se tem de 'algo', que pode ser visto num 'termômetro' e esse paradigma envolve a "esquematização do construto da 'qualidade de serviços' apresentando, situações [Confiabilidade, Tangibilidade, Sensibilidade, Segurança e Empatia] em que podem ocorrer uma lacuna entre as expectativas e percepções da clientela" (CAMPOS; MIGUEL; CARVALHO, 2018, p. 87).

\section{BIBLIOTECA PÚBLICA E AS BIBTRANSCOL}

A função da biblioteca pública, segundo Almeida júnior (1997) é levar informação para a sua comunidade de usuários promovendo a construção da cidadania através da leitura e possibilitando o conhecimento dos direitos sociais a todos os cidadãos.

\footnotetext{
A biblioteca pública é socialmente útil? Sem nenhuma dúvida, diriam muito [...] Alguém pode contestar ser a informação a matéria e o produto das atividades de uma biblioteca? Pode também ser contestado que a informação é poder, que a informação possibilita uma reflexão, uma revisão de valores e posicionamentos, uma nova postura em relação à sociedade? Em não havendo contestação, podemos afirmar que, inegavelmente, a biblioteca pública é útil socialmente [...] (ALMEIDA JÚNIOR, 1997, p. 63).
}

Rasche e Varvakis (2006, p. 138) alertam que o grande desafio para os gestores da biblioteca pública reside na capacidade de implantar serviços. Os autores também apontam

\begin{tabular}{l|l|l} 
v.16 & n.3 \\
\hline
\end{tabular}
p.408-426 set./dez. 2018 
que a "biblioteca pública, em situação de pouca utilidade, diz-nos que há algo errado, principalmente tendo em vista que a informação e o conhecimento cada vez mais são elementos indispensáveis para a participação das pessoas na sociedade [...]" (RASCHE; VARVAKIS, 2006, p. 138).

Evidencia-se que, no contexto social como um todo é preciso que "políticas públicas sejam formuladas realmente com o intuito de aportar recursos financeiros e condições materiais para que as bibliotecas funcionem com qualidade" (FERRAZ, 2014, p. 29). Por conseguinte, Almeida Junior (1997) argui que a biblioteca pública surge a serviço das causas sociais; e assim, deve ser reflexo e causa de transformações da sociedade e deve receber influência, interferir, ser início, meio e fim das alterações sociais.

Moro, Estabel e Behr (2014, p. 59) apontam que "os profissionais que atuam na biblioteca e os usuários compartilham e constroem a biblioteca juntos, em um contínuo processo de colaboração e de cooperação". Convém lembrar que a "biblioteca se transforma em um espaço democrático e personalizado por meio da prestação de serviços de qualidade que buscam satisfazer o usuário, permitindo que este se integre ao espaço e crie vínculos que despertem o sentimento de pertença" (MORO; ESTABEL; BEHR, 2014, p. 59).

Lancaster (1996) considera a biblioteca um organismo em 'crescimento', aliás, uma das cinco leis de Ranganathan (2009). Assim, ratifica-se que a biblioteca pública "desenvolve, no século XXI, [um] papel fundamental para a participação social, articulação cidadã, além de se configurar como um equipamento cultural essencial na vida cultural dos municípios brasileiros" (FERRAZ, 2014, p. 29).

Alinhada com essa perspectiva, a BibTranscol está inserida na ação governamental do Estado intitulada 'Leia Espírito Santo' e surgiu com o designo de "abrir novos capítulos na vida de quem viaja de ônibus". Esse é um projeto do Estado do Espírito Santo, da Divisão Itinerante da Biblioteca Pública do Espírito Santo (BPES) que entra na agenda governamental em prol de melhorias das áreas de alta vulnerabilidade social (que muitas vezes são demarcadas por uma espiral de violências urbana).

\footnotetext{
Atualmente, a BibTranscol atende à população que circula diariamente em todos os terminais de ônibus do Sistema Transcol composto por 10 terminais rodoviários [...] O número de pessoas que usam os Sistema Transcol mensalmente está em torno de 15.506.463 de usuários somados os 10 terminais, sendo uma média de 1.550 .646 por terminal. O modelo evoluiu para estações climatizadas, com acesso a bibliotecas digitais [...] (MAPA CULTURAL, 2017, p. 1).
}

"Com a Biblioteca Transcol, os passageiros estão a minutos de qualquer destino" - esse é o lema estampado nas portas dessa instituição e se faz presente nos dez terminais (Quadro 1) do Sistema Integrado de Transporte Coletivo Urbano Municipal e Intermunicipal de Passageiros (TRANSCOL) da Região Metropolitana da Grande Vitória (RMGV). 
Quadro 1. Rede de BibTranscol na Região Metropolitana da Grande Vitória (RMGV)

\begin{tabular}{|c|c|c|c|c|}
\hline \multicolumn{5}{|c|}{ Projeto BibTranscol } \\
\hline \multirow{11}{*}{ 莺 } & Local & Terminal de instalação & Criação & Início das atividade \\
\hline & \multirow{3}{*}{ Cariacica } & Itacibá & $3^{\circ}$ módulo & 2008 \\
\hline & & Campo Grande & $4^{\circ}$ módulo & 2009 \\
\hline & & Jardim América & $5^{\circ}$ módulo & 2009 \\
\hline & \multirow{3}{*}{ Serra } & Carapina & $8^{\circ}$ módulo & 2010 \\
\hline & & Laranjeiras & $1^{\circ}$ módulo & 2007 \\
\hline & & Jacaraípe & $10^{\circ}$ módulo & 2011 \\
\hline & \multirow{4}{*}{ Vila Velha } & São Torquato & $6^{\circ}$ módulo & 2010 \\
\hline & & Ibes & $2^{\circ}$ módulo & 2008 \\
\hline & & Itaparica & $7^{\circ}$ módulo & 2010 \\
\hline & & Vila Velha & $9^{\circ}$ módulo & 2011 \\
\hline
\end{tabular}

Fonte: Elaborado pelos autores, com base em Miguel \& Silveira (2017, p. 2029).

Cabe salientar que a primeira unidade BibTranscol foi instalada em 2007 no Terminal de Laranjeiras no município de Serra. No inicio o modelo de biblioteca era duas portas expositoras, em forma de 'banca de jornal'. Tempo depois, o modelo é adaptado para contêiner até chegar a atual estrutura de estações climatizadas; nesse ínterim de uma década, o projeto passou por diversas adequações gerenciais, estruturais e contingências até firma-se como principal ação da BPES.

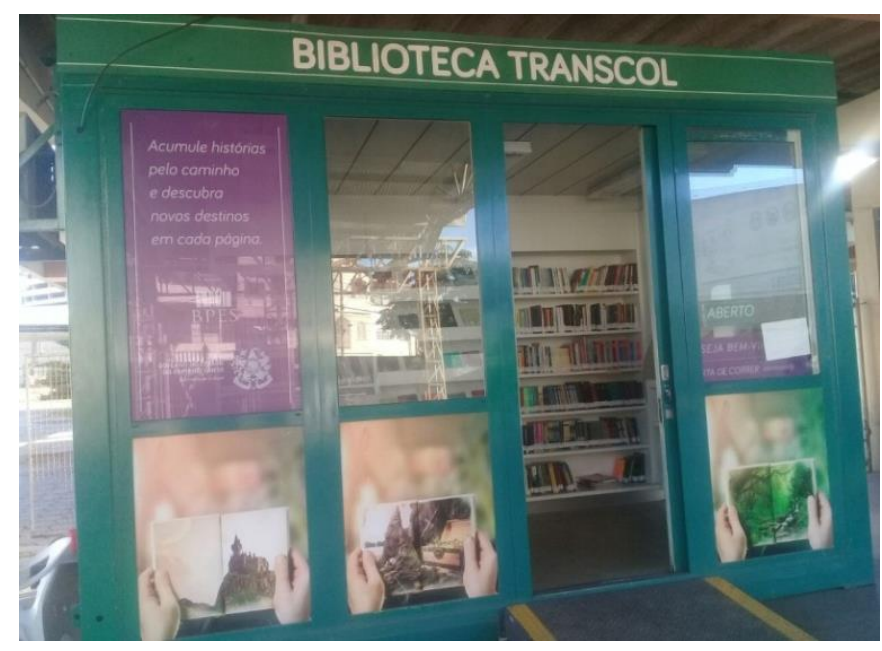

Figura 1. BibTranscol Vila Velha - Terminal Clementino Barcelos Filho Fonte: arquivo pessoal, Vila Velha, 2017

No município de Cariacica há três unidades, inseridas no terminal de Campo Grande (Antário Filho), Itacibá (Aldo Alves Prudêncio) e Jardim América (Euclério de Azevedo Sampaio); na cidade de Vila Velha há quatro módulos, abrangendo a plataforma do Ibes (Paulo Sobrinho), Itaparica (Juiz Alexandre Martins de Castro Filho), São Torquato (Floriano Rangel Mendonça) e Vila Velha (Clementino Barcelos Filho); e no município da Serra estão distribuídas pelo terminal Carapina (Abedir Barbosa de Lima), Laranjeiras (José Geraldo Motta) e Jacaraípe (Deputado Edson Vargas).

\begin{tabular}{l|l} 
v.16 & n.3
\end{tabular}
p.408-426 set./dez. 2018 


\subsection{Incursão pela tríade: qualidade, avaliação e serviços}

Nos dias atuais a buscar pela qualidade remete a um fator de sobrevivência para as organizações, isto é, em sentido conotativo, a arte de "ver a floresta e as árvores" (SENGE, 2009, p. 42). As definições para a "qualidade em serviços" mais encontrada na literatura, em geral, envolve a capacidade das organizações em satisfazer às necessidades da clientela (OLIVEIRA; MOTTA, 2013)

Rey Martín (2000, p. 153) considera que os estudos de satisfação do usuário não são um mero indicador de desempenho, mas compreendem a um instrumento sinalizador da qualidade institucional, permitindo aos gestores "antecipar ao constante processo de mudança do mundo da biblioteca e da informação" (apud ARTIGAS, 2016, p.103).

E no que tange a dificuldade da avaliação da qualidade dos serviços, Mello et al (2010, p. 9) esclarece que devido "à intangibilidade, medir a qualidade de um serviço é frequentemente muito difícil. A medição normalmente é feita através da percepção do usuário sobre o serviço prestado e a expectativa do serviço que ele esperava receber". Sob o ponto de vista do marketing, Oliveira e Motta (2013, p. 140) reportam que a "qualidade é uma variável complexa e resulta da relação entre diversas variáveis simples. Visto de outra forma, qualidade é um construto etéreo [...]". Por conseguinte, os autores apontam que "a qualidade é um conceito relativo e não absoluto" e assim, expressa a satisfação das necessidades.

Os autores acima citados entendem que atualmente a 'filosofia da qualidade' sofre uma transfiguração e, nesse sentido, o termo 'qualidade' vem sendo "substituída por 'valor'. Valor diz respeito também à satisfação de necessidades, ou seja, produtos que têm valor são aqueles capazes de satisfazer as necessidades dos consumidores aos quais são destinados. No entanto, 'valor' é um construto mais completo que 'qualidade' [...]" (OLIVEIRA; MOTTA, 2013, p. 140).

A qualidade percebida tem sido definida como uma relação entre as percepções (importância, qualidade desejada) e as Expectativas (performance percebida, desempenho) de um serviço (PARASURAMAN; ZEITHAML; BERRY, 1985) e, pode ser mensurada tomando como base a possibilidade de que o interagente não tem um único nível de expectativa para cada determinante mensurada e que a qualidade de serviço e a satisfação de serviço são construtos diferentes, mas que se relacionam (RODRIGUES, 2000).

\footnotetext{
A importância da qualidade em serviço é tal que o assunto se coloca como um dos mais discutidos na literatura de marketing, uma vez que se busca, não apenas um consenso ao seu respeito, mas, principalmente, maneiras de organizá-la e transmitila adequadamente[...] A percepção de qualidade pode ser considerada uma função do que o cliente obteve em relação às expectativas, do que esperava obter, ou seja, diz respeito ao grau de dissonância entre a expectativa e o obtido [...] (OLIVEIRA; 2013, p. 153).
} 
Para Fitzsimmons e Fitzsimmons (2005) o aspecto essencial da prestação de serviço é a compreensão de que o cliente pode ser uma parte ativa do processo. E de maneira simplificada, Las Casas (2017, p. 6) esclarece que "é importante observar que os serviços possuem dois componentes de qualidade que devem ser considerados: o serviço propriamente dito e a forma como é percebido pelo cliente".

Rozados (2004) assinala que sejam quais forem as necessidades ou as particularidades de cada organização, a avaliação de serviços é essencial para o planejamento, pois auxilia na tomada de decisão e, principalmente, na gestão da qualidade. Porque sem qualidade corre-se o risco de perder o cliente, e por isso são importantes os diagnósticos. A avaliação da qualidade dos serviços, de acordo com Las Casas (2017, p. 215), permite que as organizações obtenham conhecimento acerca "das percepções, reações e atitudes dos seus clientes em relação à entrega dos serviços. Os serviços podem ser avaliados em termos de várias características ou dimensões".

Em suma, o "ideal na avaliação da qualidade de serviços compreende em igualar os quocientes da expectativa dos clientes com a percepção dos mesmos, ou seja, que a diferença entre eles seja nula ou com pouca variação" (SOARES, SOUSA, 2015, p. 84). Conforme aponta Lancaster (1996, p. 1), "uma avaliação é feita não como um exercício intelectual, mas para reunir dados úteis para atividades destinadas a solucionar problemas ou tomar decisões". Sendo assim, uma "avaliação é um elemento essencial da administração bem sucedida de qualquer empreendimento" (LANCASTER, 1996, p. 15).

Lancaster (1996) assinala quatro motivos norteadores para se pensar na necessidade de avaliação dos serviços na esfera de serviços bibliotecários e assim, aponta que: (I) faz-se necessário estabelecer uma escala para mostrar em que nível de desempenho o serviço está funcionando no momento; (II) avaliar serve para comparar o desempenho de várias bibliotecas ou serviços; (III) o diagnóstico serve para justificar de forma clara a existência da instituição; e por fim (IV) com o resultado é identificar possíveis deficiências e/ou a ineficiência dos serviços. O autor ressalta que a avaliação de um serviço de informação "[...] será um exercício estéril caso não seja conduzida com o objetivo específico de identificar maneiras de melhorar seu desempenho" (LANCASTER,1996, p. 8).

\subsection{O instrumento Servqual}

Parasuraman, Zeithaml e Berry (1985) instrumentalizaram a escala Servqual de múltiplos itens para avaliar a qualidade dos serviços explorando a construção de uma métrica (multiponto) utilizada para análise geral da qualidade dos serviços prestados, e tal estrutura de avaliação é embasada no uso da psicométrico de um escala do tipo Likert. Mello et al (2010), Oliveira (2013) e Las Casas (2017) reportam que a abordagem Servqual é uma escala concisa de múltiplos itens com boa confiabilidade e validade, revelando-se um 'Qualitômetro' adequado para avaliar a satisfação dos clientes, uma vez que agrupa os construtos

\begin{tabular}{|c|c|c|c|c|c|}
\hline$C I \cdot R \rho, D i$ & Campinas, SP & v.16 & n. 3 & p. 408-426 & \\
\hline
\end{tabular}


determinantes da qualidade e sua relação com a satisfação.

A dimensão da confiabilidade consiste na capacidade de realizar o que é prometido ao cliente e padronização no fornecimento de serviço; a determinante tangibilidade (ou tangíveis) consiste na aparência, as representações físicas, ou seja, elementos físicos como equipamentos, instalações, pessoal e material de comunicação; a dimensão sensibilidade (transcrita algumas vezes como responsividade ou receptividade) envolve a disposição da empresa, a pontualidade e a agilidade no atendimento, envolve o tempo de espera pelo serviço, a determinante segurança (também assinada como garantia) está relacionada à baixa percepção de risco pelo cliente, sendo que estes aspectos são transmitidos pelos funcionários por meio do conhecimento e cortesia, juntamente com a competência da instituição e a segurança das intervenções; a dimensão da empatia envolve a atenção personalizada e cuidados individuais, no qual o cliente tem a sensação de que são especiais para organização, possibilitando o fornecimento de informações adequadas às demandas do cliente e maior comunicação (BERRY; PARASURAMAN, 1992).

O modo como os usuários percebem essas cinco dimensões/determinantes da qualidade define se os clientes tiveram uma experiência com um serviço de qualidade e se estão satisfeitos com o serviço prestado. Afinal, o principal objetivo de uma avaliação de serviços é melhorar os serviços com base na opinião e necessidades dos usuários (MIGUEL, 2017a).

\section{MÉTODO}

A exigência por serviços de qualidade em unidades de informação (arquivos, bibliotecas, museus, centros de documentação) remete a uma busca permanente por instrumentos que possam avaliar o nível de satisfação dos clientes. $\mathrm{O}$ modelo apresentado neste estudo é uma adaptação da ferramenta Servqual para biblioteca pública.

Este estudo possui caráter descritivo de natureza quantitativa e a amostra adotada foi do tipo não probabilística por conveniência. Seu delineamento é do tipo levantamento (survey) com a utilização de questionário semiestruturado disponibilizado entre os dias 23 janeiro de 2017 a 24 de março de 2017, sendo o instrumento aplicado com o intuito de medir e comparar o grau de importância (expectativas; qualidade esperada) com a satisfação do cliente (percepção; qualidade percebida). $\mathrm{O}$ instrumento foi aplicado após o/a cliente sair do módulo BibTranscol, pois segundo a abordagem teóricametodológica Servqual reporta que somente pode avaliar quem de fato utilizou (teve contato) com o serviço.

O pré-teste foi com amostra de 24 associados da BibTranscol de Itaparica, no mês de novembro de 2016, com o objetivo de averiguar a clareza textual do questionário e o tempo médio de resposta, obtendo-se um tempo médio de 7 minutos. O pré-teste teve como finalidade de ratificar a eficácia do instrumento de coleta de dados em situações reais de coleta, conforme recomenda Gil (2002), Hair (2009) e Lakatos \& Marconi (2010). 
Para a coleta de dados utilizou-se a abordagem teórico-metodológica Servqual, com as dimensões ou determinantes da qualidade adaptadas à realidade em estudo e aplicado aos associados (de 18 anos ou mais). O roteiro de perguntas possuía três perguntas sociométricas (faixa etária, etnia e estado civil) e vinte e dois pares de sentenças de opinião, elaboradas e distribuídas entre as cinco dimensões da qualidade para avaliar o grau de 'percepção' e 'expectativas' diante a ordem escalar de Likert.

Optou-se pela Likert de 5 alternativas (outras podem representar entre 4 e 10 itens) correspondendo aos conceitos discordo totalmente (1), discordo (2), indiferente (3), concordo (4) e concordo totalmente (5) pois foi a que melhor se adequou na fase de pré-teste de instrumento.

Depois de concluir a coleta de dados, foi elaborado um banco de dados com o levantamento e, os resultados foram tabulados para posteriormente análise (estatística descritiva).

\section{TABULAÇÃo E ANÁLISE DE DADOS}

Um total de 440 questionários foram aplicados a população estabelecida em estudo e, foram considerados para análise os que tinham a resposta 'Sim' à questão: 'Utilizei os serviços da BibTranscol' e, portanto, validou-se 420 questionários $(95,45 \%)$ e assim, obtivese 105 em cada biblioteca da rede. "Convêm relembrar que aplicação da abordagem teóricometodológica Servqual requer uma prévia experiência com o serviço a ser avaliado" (MIGUEL, 2017b, p. 255).

A coleta de dados foi aleatória e direcionada aos transeuntes que utilizaram o serviço. A amostra apresentou um equilíbrio entre os sexos, sendo que 53,57\% do total são do sexo feminino e 46,43\% masculino, conforme demonstrado na tabela 1.

Tabela 1. Perfil social dos participantes

\begin{tabular}{|c|c|c|c|c|c|c|c|c|c|c|c|c|}
\hline \multicolumn{13}{|c|}{ PERFIL SOCIAL } \\
\hline \multirow{2}{*}{ Módulo } & \multicolumn{3}{|c|}{ Terminal do Ibes } & \multicolumn{3}{|c|}{ T. São Torquato } & \multicolumn{3}{|c|}{ T. Vila Velha } & \multicolumn{3}{|c|}{ T. Itaparica } \\
\hline & $\begin{array}{l}\text { n. } 50 \\
\text { masc. }\end{array}$ & $\begin{array}{l}\text { n. } 55 \\
\text { fem. }\end{array}$ & $\begin{array}{c}\text { Total } \\
\%\end{array}$ & $\begin{array}{l}\text { n. } 48 \\
\text { masc. }\end{array}$ & $\begin{array}{l}\text { n. } 57 \\
\text { fem. }\end{array}$ & $\begin{array}{c}\text { Total } \\
\%\end{array}$ & $\begin{array}{l}\text { n. } 45 \\
\text { masc. }\end{array}$ & $\begin{array}{l}\text { n. } 60 \\
\text { fem. }\end{array}$ & $\begin{array}{c}\text { Total } \\
\%\end{array}$ & $\begin{array}{l}\text { n. } 52 \\
\text { masc. }\end{array}$ & $\begin{array}{l}\text { n. } 53 \\
\text { fem. }\end{array}$ & $\begin{array}{c}\text { Total } \\
\%\end{array}$ \\
\hline$<30$ & 31 & 35 & $63 \%$ & 26 & 30 & $53 \%$ & 22 & 37 & $56 \%$ & 31 & 30 & $58 \%$ \\
\hline $30-60$ & 17 & 15 & $30 \%$ & 18 & 20 & $36 \%$ & 19 & 23 & $40 \%$ & 19 & 22 & $39 \%$ \\
\hline$>60$ & 2 & 5 & $7 \%$ & 4 & 7 & $10 \%$ & 4 & 0 & $4 \%$ & 2 & 1 & $3 \%$ \\
\hline \multicolumn{13}{|l|}{ Etnia } \\
\hline Branco & 33 & 37 & $67 \%$ & 27 & 27 & $51 \%$ & 26 & 31 & $54 \%$ & 30 & 20 & $48 \%$ \\
\hline Pardo/Negro & 14 & 17 & $30 \%$ & 17 & 29 & $44 \%$ & 14 & 26 & $38 \%$ & 17 & 32 & $47 \%$ \\
\hline Outro (a) & 3 & 1 & $4 \%$ & 4 & 1 & $5 \%$ & 5 & 3 & $8 \%$ & 5 & 1 & $6 \%$ \\
\hline \multicolumn{13}{|l|}{ Estado civil } \\
\hline (C) RDBCI: Rev & is $D$ & & & $\overline{\mathrm{C}}$ & 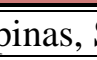 & & & & $\overline{08-}$ & & $1 \mathrm{de}$ & $\overline{18}$ \\
\hline
\end{tabular}




\begin{tabular}{l|l|l|l|l|l|l|l|l|l|l|l|l}
\hline $\begin{array}{l}\text { Solteiro (a) } \\
\text { Casado (a) }\end{array}$ & 35 & 34 & $66 \%$ & 27 & 32 & $56 \%$ & 32 & 34 & $63 \%$ & 32 & 41 & $70 \%$ \\
\hline \begin{tabular}{l} 
Outro (a) \\
\hline
\end{tabular} & 5 & 7 & $23 \%$ & 19 & 17 & $34 \%$ & 8 & 23 & $30 \%$ & 11 & 11 & $21 \%$ \\
\hline
\end{tabular}

Fonte: pesquisa de campo

No grupo feminino, a maioria se encontrava com menos de 30 anos (58,67\%), declararam-se brancas $(51,11 \%)$ e solteiras $(62,67 \%)$. Entre os homens, a maioria assinalou faixa etária de 30 anos ou menos $(56,41 \%)$, declararam-se de etnia branca $(59,49 \%)$ e solteiros $(64,62 \%)$.

\subsection{Expectativas e percepção}

Em relação as expectativas dos clientes e percepções quanto aos serviços prestados pelas BibTranscol, identificamos que o item mais importante na opinião dos participantes foi "Facilidade dos funcionários em se comunicar", com 88,10\% das respostas, desafortunadamente também foi este o indicador com a menor satisfação, com apenas $27,14 \%$ das respostas marcando a opção referente a "muito satisfeito". O segundo de expectativa mais elevada é atribuída a questão "Funcionários acessíveis e gentis", com $85,71 \%$ das respostas. No tocante a essa esfera da expectativa, esses dois itens obtiveram dos associados em média uma elevada pontuação (entre 3,90 e 4,80 em uma escala de cinco pontos), o que indica que os fatores selecionados para a pesquisa são realmente relevantes para comunidade usuária.

Entre os itens apontados como de "maior percepção", o primeiro deles, com 71,90\% das respostas foi "Funcionários têm conhecimento para responder as questões", e o segundo, com $60,71 \%$, "Instalações físicas com visual atraente". E reportando a esfera da satisfação, os interagentes atribuíram às questões em estudo, uma pontuação média variante entre 3,65 e 4,60 , apontando em todos os 22 itens avaliativos uma pequena diferença entre a expectativa e o desempenho dos serviços.

A tabela 2 apresenta a média da pontuação recebida pelos indicadores da qualidade de serviço a respeito das métricas expectativas e percepções, agrupados por dimensão:

Tabela 2. Expectativas e Percepção por Dimensão (em uma escala de 1 a 5)

\begin{tabular}{cc|c|c|c|c|c|c}
\hline \multirow{2}{*}{ Dimensões } & \multicolumn{2}{c|}{ Expectativas (Importância) } & \multicolumn{2}{c|}{ Percepções (Satisfação) } & \multicolumn{2}{c}{ Gap (Lacuna) } \\
\cline { 2 - 8 } & Masculino & Feminino & Média & Masculino & Feminino & Média & $\mid$ Média $\mid$ \\
\hline Confiabilidade & 4,70 & 4,60 & 4,65 & 4,30 & 4,30 & 4,30 & 0,35 \\
\hline Tangibilidade & 4,30 & 4,70 & 4,51 & 4,05 & 4,20 & 4,13 & 0,38 \\
\hline Sensibilidade & 3,90 & 4,40 & 4,17 & 3,65 & 3,65 & 3,65 & 0,52 \\
\hline Segurança & 4,80 & 4,80 & 4,80 & 4,60 & 4,55 & 4,57 & 0,23 \\
\hline Empatia & 4,30 & 4,50 & 4,41 & 3,75 & 3,60 & 3,67 & 0,74 \\
\hline
\end{tabular}

Fonte: pesquisa de campo

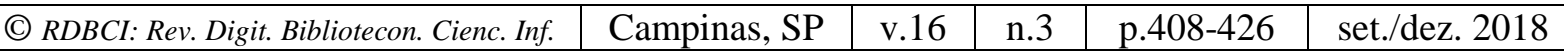


As determinantes da qualidade também podem ser analisadas sobre a ótica da identidade de gênero; o painel apresentado pela tabela 2 mostra uma paridade quando pensamos na dimensão Segurança (assurance) a qual obteve média de 4,80; e isso indica que tal determinante é a preferida pelos respondentes que utilizam alguma BibTranscol em itens de satisfação mais elevados $(4,57)$ apresenta-se condizendo a um bom desempenho.

\subsection{Análise de Quadrantes e de Gaps}

Nitecki e Hernon (2000) recomendam a Análise de Quadrante (matriz importância e desempenho), que consiste em uma correlação gráfica que facilita a visualização das informações (em quatro áreas), compreendendo, uma ferramenta teórico-metodológica para a estratégia organizacional.

A análise da matriz se constrói pela função bidimensional em que as expectativas (importância; desejos) é mostrada pelo 'eixo x' e, se atribui ao 'eixo y' as Percepções (satisfação; necessidades). Considerado a ilustração a seguir, o posicionamento dos 22 atributos da qualidade sinalizam por exemplo, que um item situado no Quadrante I têm alta importância e um alto desempenho (satisfação) e isso representa uma possível vantagem competitiva organizacional.

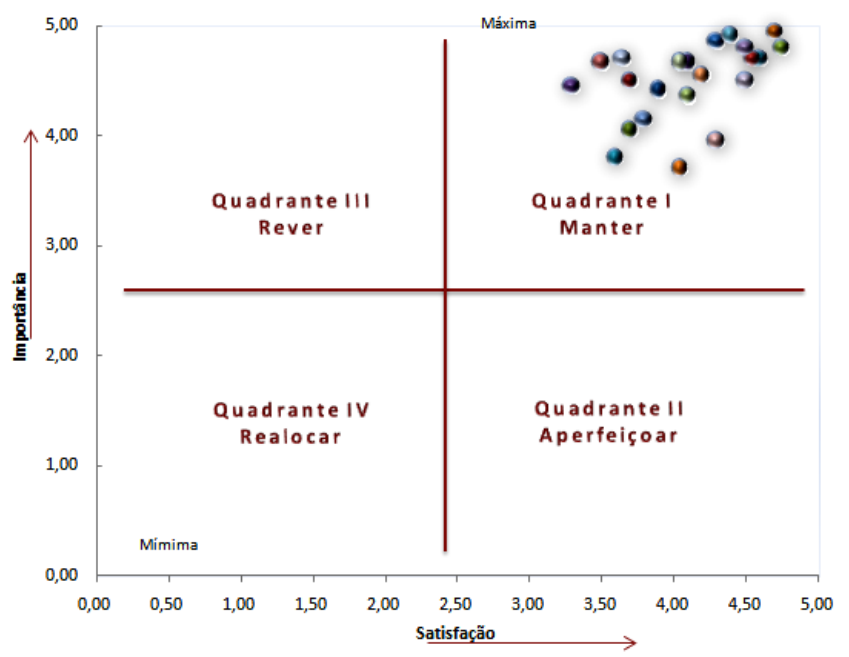

Figura 2. Análise de Quadrantes Dimensões da 'Qualidade em Serviços' Fonte: Dados da pesquisa (2017)

Nessa pesquisa houve uma concentração no quadrante 1 (manter) de todos os vinte e dois atributos da qualidade e, isso sugere que os associados veem todos esses atributos como sendo altamente importantes e bem desempenhados pelas BibTranscol de Vila Velha.

Outro possível esquema de interpretação dos dados e, largamente utilizado em pesquisa é conhecido como 'Análise de Gaps'. O modelo gap da qualidade corresponde a 
abordagem necessária para projetar os dados coletados e classificar a qualidade dos serviços conforme a avaliação dos interagentes acionados.

O formato de duas secções do instrumento de coleta de dados, demarcando duas zonas (expectativas e às percepções) de sentidos demonstra que Servqual oferta eficácia métrica para apurar o construto da qualidade. Miguel e Freire (2016, p. 112) apontam que a análise de gaps é uma boa maneira para apresentar os resultados da pesquisa e, o cálculo mediante a aplicação da seguinte fórmula: "Gap = Satisfação - Importância".

Para calcular gap model as médias, as pontuações (de 1 a 5) de cada assertiva foram somadas e divididas pelo número total de respostas (420, eliminado 20 que assinalaram não ter utilizado o serviço/atendimento), tanto para o quesito Percepção como para o quesito Expectativa; a seguir foi calculada a diferença entre as duas diretrizes de análise, obtendo-se assim, o gap por questão. "Funcionários têm conhecimento adequado para atendê-lo", obteve o menor gap da pesquisa, ou seja, 0,10; ao contrário de "Funcionários entendem suas necessidades específicas", cujo gap foi calculado em 0,95 , o maior hiato entre os serviços adicionados na pesquisa.

Nitecki e Hernon (2000) assinalam em seus estudos que as pontuações de gaps entre 0 e -1 não superam as expectativas (excelência), contudo sinalizam se o desempenho do serviço vai ao encontro dos anseios da comunidade usuária, pode-se atender (Figura 3) que os interagentes das BibTranscol percebem um serviço padrão e, auferem um tom de qualidade em perspectivas positiva ao serviço prestado pela rede bibliotecária.

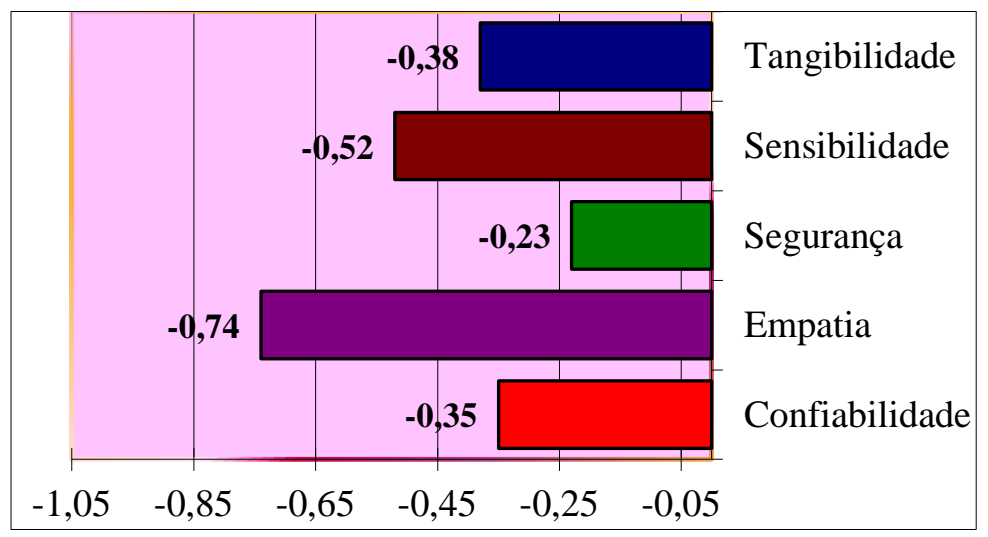

Figura 3. Gráfico dos gaps por dimensões

Fonte: o(s) autor(es); pesquisa de campo

Pode-se averiguar que os hiatos ente o 'serviço desejado' e o de 'fato encontrado' abrange em essencial a dimensão 'empatia' $(|0,74|)$ e a 'sensibilidade' $(|0,52|)$. Os interagentes desse estudo apontaram que há um descuido com as esferas receptividade e empatia; essa remete "a atenção e o carinho individualizados proporcionados aos clientes" (BERRY; PARASURAMAN, 1992, p.30) e; aquela, expressa "a disposição para ajudar o cliente e proporcionar com presteza um serviço" (1992, p.30).

\begin{tabular}{l|l|l|l|l|l}
\hline (C) RDBCI: Rev. Digit. Bibliotecon. Cienc. Inf. & Campinas, SP & v.16 & n.3 & p.408-426 & set./dez. 2018 \\
\hline
\end{tabular}


Diante essa acepção e considerando os achados apontando que a dimensões de segurança $(4,57)$ e confiabilidade $(4,30)$ representaram os atributos com maior grau de satisfação e que nas dimensões de sensibilidade $(3,65)$ e empatia $(3,67)$ estavam inseridos os atributos com menores níveis de satisfação, verifica-se portanto que a pesquisa reitera a importância dos serviços biblioteconômicos contarem com instrumentos de avaliação que consintam a seus gestores planejar melhorias e apontarem a necessidade de adequar o processo de desenvolvimento de serviços.

Nesse sentido, Las Casas (2017, p. 189) alude que "a empatia, a capacidade de se colocar no lugar dos outros é o respeito ao cliente. Saber onde se pode ir. Considerá-lo como ser humano, valorizá-lo dentro dos princípios básicos da moral humana" (2017, p. 189) e somente assim, conquistando a admiração e o cuidado com o ser que a qualidade total poderá ser alcançada.

\section{CONSIDERAÇÕES FINAIS}

A metodologia utilizada nesta pesquisa, por meio da aplicação de uma adaptação do modelo Servqual torna viável obter uma mensuração geral da qualidade dos serviços por meio da média da pontuação obtida entre o grau de percepção e o grau de expectativa. E justamente o olhar dos passageiros de transporte coletivo de Vila Velha que direciona essa análise sobre a qualidade dos serviços prestados por quatro Bibtranscol (no bairro Ibes, terminal Paulo Sobrinho; em Itaparica, terminal Juiz Alexandre Martins de Castro Filho; em São Torquato, terminal Floriano Rangel Mendonça; e a instalada no terminal de Vila Velha; Clementino Barcelos Filho).

É de extrema importância o conhecimento das expectativas e das percepções de qualidade dos serviços prestados, pois possibilita que as unidades de informação tenham um indicador detalhado em cada ambiência organizacional. Estas informações viabilizam a projeção de vindouras mudanças e melhorias no processo de desenvolvimento de serviços com o objetivo de conquistar e reter interagentes reais e potenciais, diversificar ações e mudar a realidade.

Os resultados demonstraram que o objetivo do estudo foi atingido, o modelo adaptado Servqual torna-se um instrumento apropriado para medir a qualidade dos serviços prestados a partir da perspectiva dos clientes da biblioteca pública (BibTranscol). Ao encontro do que aponta Almeida Júnior (1997, p. 77), compreendemos que muitas vezes "a visão que se tem da biblioteca é, normalmente, de algo estático, pacato, sem dinamismo. Mas, a biblioteca pode e deve ser exatamente o contrário".

Por meio dessa pesquisa foi possível constatar que os respondentes percebem positivamente a qualidade, mas há descuidos da instituição com a questão empática da

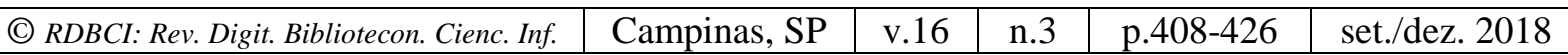


qualidade. Advém registrar que o agravamento das lacunas gera insatisfação e, o cálculo dos gaps por dimensões apontam as maiores discrepâncias entres expectativas e percepções (gap 0,74). Isso expressa um ponto fraco, demonstrando a necessidade de se investir em capacitações e treinamentos, em prumo a uma política que promova o aperfeiçoamento contínuo da equipe, juntamente incluindo o profissional bibliotecário para acompanhar os planos e ações.

Reiteramos que os clientes atendidos pela equipe estão satisfeitos com o atendimento prestado pela Biblioteca Transcol, citando a Segurança (garantia) como uma das mais importantes $(4,80)$. A pesquisa ratifica que a métrica Servqual (com as devidas adaptações) é apropriada para analisar a qualidade a partir das percepções e expectativas da comunidade usuária dos serviços.

As desvantagens do modelo padrão remete a um questionário composto apenas por perguntas fechadas, inviabiliza aos interagentes expressarem opiniões, críticas e sugestões essenciais ao diagnóstico. Para minimizar este problema, sugere-se a inclusão de um campo para resgatar apreensões, sugestões e críticas. Por conseguinte a adoção do modelo depende do tipo de avaliação a ser desenvolvida, e de ajustes necessários a ambiência pesquisada, o que pode ser implementado um questionário adequado pela incrementa advindas de abordagens qualitativas.

Considera-se a limitação da pesquisa em termos da impossibilidade de realizar generalizações, a adoção de uma amostra não probabilística impossibilita generalização sobre os achados empíricos para a população de estudo. Outro dilema envolve o aprofundamento da interpretação de dados pelo painel da identidade de gêneros, que é um tema que envolve pontos nevrálgicos da ética em pesquisa aplicadas. Para estudos futuros, sugere-se um refinamento dos métodos estatísticos (jus a robusta credibilidade e versatilidade da métrica), uma vez que não se busca incluir no processo de desenvolvimento de serviços diferentes maneiras de organizar e transmitir valor a gestão de alto padrão.

Registra-se ainda, através do que foi exposto, que todas as organizações, não só as BibTrancol, mas as instituições em geral devem considerar que as expectativas de qualidade da comunidade usuária dos serviços e que estas expectativas são determinísticas na percepção do valor dos seus serviços. Assim, muitas são as possibilidades e destinos... "se o século XIX caracterizou-se pela construção de bibliotecas, o século XX ficou marcado por sua destruição" (BATTLES, 2003, p. 157) e o presente século é pautado em sua transformação, superação e viva construção de possibilidades.

\section{REFERÊNCIAS}

ALMEIDA JÚNIOR, O. F. de. Sociedade e biblioteconomia. São Paulo: Polis, 1997. 129p.

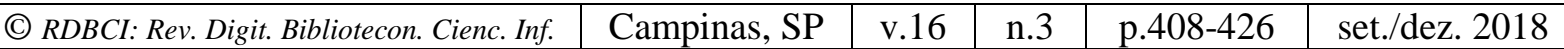


ARTIGAS, C. M. T. Bibliotecas de centros de pesquisa no século XXI: desafios e perspectivas. In: RIBEIRO, A. C. M. L.; FERREIRA, P. C. G. (Org.). Biblioteca do século XXI: desafios e perspectivas. Brasília: Ipea, 2016. p: 95-113. Disponível em: <http://www.ipea.gov.br/agencia/images/stories/PDFs/livros/livros/170105_biblioteca_do_se culo_21.pdf $>$. Acesso em: 11 nov. 2017.

BARGANHA, F. Novas Bibliotecas, Novos Conceitos. Revista da Faculdade de Ciências Humanas e Sociais, Porto, Universidade Fernando Pessoa: UFP, n. 1, 2004, p.93-97. Disponível em: <http://hdl.handle.net/10284/616>. Acesso em: 19 dez. 2016.

BATTLES, M. A conturbada história das bibliotecas. São Paulo: Planeta do Brasil, 2003. $238 \mathrm{p}$.

BERRY, L. L.; PARASURAMAN, A. Serviços de marketing: competindo através da qualidade. São Paulo: Maltese: Norma, c1992. 238 p.

CAMPOS, A. C. B.; MIGUEL, M. C.; CARVALHO, S. M. S. de. Aplicação do modelo Servqual na bienal do livro da Zona da Mata: o olhar dos interagentes abrindo novas páginas para melhoria da qualidade. Encontros Bibli: revista eletrônica de biblioteconomia e ciência da informação, Florianópolis, v. 23, n. 52, p. 84-94, maio 2018. ISSN 1518-2924. Disponível em: <https://periodicos.ufsc.br/index.php/eb/article/view/15182924.2017v23n52p84/36461>. Acesso em: 29 maio 2018. doi:https://doi.org/10.5007/1518$2924.2017 \mathrm{v} 23 \mathrm{n} 52 \mathrm{p} 84$.

FERRAZ, M. N. O papel social das bibliotecas públicas no século xxi e o caso da superintendência de bibliotecas públicas de minas gerais. Perspectivas em Ciência da Informação, v. 19, 2014. Disponível em: <http://www.brapci.inf.br/v/a/17845>. Acesso em: 29 Abr. 2018.

FITZSIMMONS, J. A.; FITZSIMMONS, M. J. Administração de serviços: operações, estratégia e tecnologia da informação. 4. ed. Porto Alegre: Bookman, 2005. 564 p.

FONSECA, E. N. da. Introdução á biblioteconomia. 2. ed. São Paulo: Briquet de Lemos, 2007, 152 p.

FREITAS, J.; REGEDOR, A. B. Bibliotecas públicas e cidadania activa. In: CONGRESSO NACIONAL DE BIBLIOTECÁRIOS, ARQUIVISTAS E DOCUMENTALISTAS, 2., 2007, Ponte Delgada. Anais... Ponte Delgada: BAD, 2007. p. 1-17. Disponível em: $<$ https://www.bad.pt/publicacoes/index.php/congressosbad/article/view/517/284>. Acesso em: 24 nov. 2017.

GIL, A. C. Como elaborar projetos de pesquisa. 4. ed. São Paulo: Atlas, 2002. 175 p.

HAIR, J. F. Análise multivariada de dados. 6. ed. Porto Alegre: Bookman, 2009. X, 688 p.

LAKATOS, E. M.; MARCONI, M. de A. Fundamentos de metodologia científica. 7. ed. São Paulo: Atlas, 2010. 297 p.

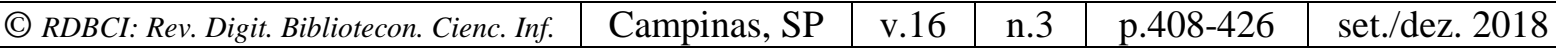


LANCASTER, F. W. Avaliação de serviços de bibliotecas. Brasília, DF: Briquet de Lemos, 1996. $356 \mathrm{p}$.

LAS CASAS, A. L. Qualidade total em serviços: conceitos, exercícios, casos práticos. 6. ed São Paulo: Atlas, 2017. 223 p.

MAPA CULTURAL [Secretaria de Estado da Cultura do Espírito Santo - SECULT; Biblioteca Pública do Espírito Santo - BPES]. Programa - Projeto BibTranscol. SECULT: BPES, Vitória, 2017. Disponível em: <http://mapacultural.es.gov.br/projeto/257/> Acesso em: 29 set. de 2016.

MELLO, C. H. P. et al. Gestão do processo de desenvolvimento de serviços. São Paulo: Atlas, 2010. VIII, 194 p.

MIGUEL, M. C. Múltiplos olhares em prol da qualidade de serviços biblioteconômicos. Revista ACB, [S.1.], v. 22, n. 2 ESPECIAL, p. 192-207, jul. 2017a. ISSN 1414-0594. Disponível em: <https://revista.acbsc.org.br/racb/article/view/1312〉. Acesso em: 29 maio 2018.

MIGUEL, M. C. Novos tempos, novos desafios: o olhar dos associados da Biblioteca Transcol. Sinais (UFES), v. 21, p. 244-265, 2017b. Disponível em: <http://periodicos.ufes.br/sinais/article/viewFile/15484/12479>. Acesso em: 29 nov. 2017.

MIGUEL, M. C; FREIRE, V. F. Avaliação da Qualidade Orientada ao Usuário do Museu Capixaba do Negro: Aplicação da Abordagem Teórico-Metodológica. Guará, Vitória, n. 05, p. 103-116, Jul, 2016. Disponível em:

<http://periodicos.ufes.br/guara/article/view/14350/10092>. Acesso em: 19 dez. 2016.

MIGUEL, M. C.; SILVEIRA, R. Z. Percepções e Expectativas dos Associados da Biblioteca Transcol em Encontro aos seus Dez Anos de Atuação. Revista Eletrônica Gestão e Serviços, v. 8, n. 2, p. 2021-2041, 2017. Disponível em: <www.metodista.br/revistas/revistas-ims/index.php/REGS/article/view/7386>. Acesso em: 17 fev. 2017.

MORO, E. L. da S.; ESTABEL, L. B.; BEHR, A. Gestão em bibliotecas. In: MORO, E. L. da S.; ESTABEL, L. B. (Org.). Biblioteca: conhecimentos e práticas. Porto Alegre: Penso, 2014, cap. 4, p. 57-76.

NITECKI, D. A.; HERNON, P. Measuring service quality at Yale's University's libraries. The Journal of Academic Librarianship, Ann Arbor, v. 26, n. 4, p. 259-273, Jul. 2000.

NUNES, M. B. Leitura, literacias e inclusão social: novos e velhos desafios para as bibliotecas públicas. In: LOPES, J. T. (Org.). Práticas de dinamização da leitura. Porto: Setepés, 2007. p: 48-59. Disponível em: $<$ http://www.setepes.pt/Imgs/Colectanea\%20de\%20Textos\%20\%20Praticas\%20de\%20Dinamizacao\%20da\%20Leitura(1).pdf>. Acesso em: 17 dez. 2017. 
OLIVEIRA, B. Estratégias e decisões de serviços. In: MATTAR, F. N. (Org.). Gerência de produtos: estratégias e ações para o sucesso. Rio de Janeiro: Elsevier, 2013. p: 145-158.

OLIVEIRA, B.; MOTTA, S. L. S. Estratégias e decisões de produtos. In: MATTAR, F. N. (Org.). Gerência de produtos: estratégias e ações para o sucesso. Rio de Janeiro: Elsevier, 2013. p: 135-144.

PARASURAMAN, A; ZEITHAML, V. A; BERRY, L. L. A conceptual model of service quality and its implications for future research. Journal of Marketing, Chicago, v. 49, n. 4, p. 41-50, 1985.

RANGANATHAN, S. R. As cinco leis da biblioteconomia. Brasília: Briquet de Lemos, 2009. xxv, $336 \mathrm{p}$.

RASCHE, F.; VARVAKIS, G. Bibliotecas públicas e seus serviços. In: CUNHA, M.; SOUZA, F. das C. de. (Org.). Comunicação, gestão e profissão: abordagens para o estudo da ciência da informação. Belo Horizonte: Autêntica, 2006. p: 127-140.

RODRIGUES, A. M. Qualidade de serviços e satisfação do consumidor: aspectos conceituais distintivos. In: ENCONTRO NACIONAL DA ASSOCIAÇÃO DOS PROGRAMAS DE POS-GRADUAÇÃO EM ADMINISTRAÇÃO, 24.,2000, Florianópolis. Anais... Rio de Janeiro: ANPAD, 2000. Disponível em: < http://docplayer.com.br/33745551-Qualidade-deservicos-e-satisfacao-do-consumidor-aspectos-conceituais-distintivos.html >. Acesso em: 02 fev. 2014.

ROZADOS, H. B. F. Indicadores como ferramentas para gestão de serviços de informação. 2004. Tese (Doutorado em Ciência da Informação). Disponível em: <http://www.lume.ufrgs.br/bitstream/handle/10183/5668/000429419.pdf>. Acesso em: 04 fev. 2013.

SENGE, P. M. A quinta disciplina: arte e prática da organização que aprende. 25. ed. Rio de Janeiro: Best Seller, 2009. 530 p.

SOARES, L. M. F.; SOUSA, C. V. Percepção da qualidade de serviços nas bibliotecas da Universidade Federal de Ouro Preto na perspectiva do usuário. Perspectivas em Ciência da Informação, [S.1.], v. 20, n. 2, p. 79-99, jun. 2015. Disponível em: <http://portaldeperiodicos.eci.ufmg.br/index.php/pci/article/view/2050>. Acesso em: 25 jan. 2018 . 


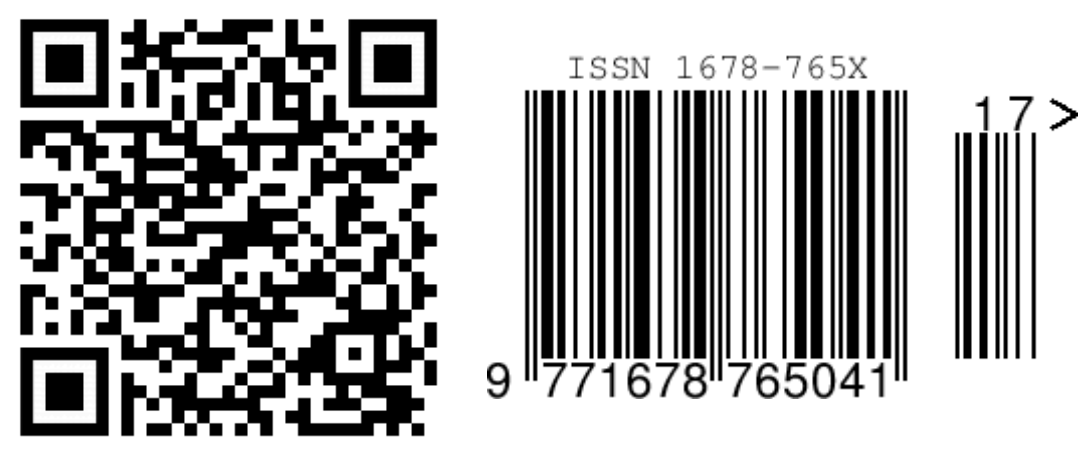

